\title{
THE DENTIFRICE PROBLEM
}

\author{
By WILliaM J. GIES,1 New York City
}

(Read before the Illinois State Dental Society, Peoria, Illinois, May 10-12, 1921)

$\mathrm{T}$ WELVE years ago Dr. J. Morgan Howe, of New York City, asked me to co-operate with the local dental society in the study of salivary anomalies and their possible relation to dental caries. I was, then, I believe, like a fairly typical member of the average medical faculty-I felt that "tecth were teeth," more or less to be ignored from the dynamic standpoint, not at all interesting physiologically, and never figuring importantly in serious physiological considerations. I remember I was much impressed by the fact that Dr. Howe, a man of about seventy, appealed to me, a relatively young man. I was more impressed by that than by his proposal itself. We began, promptly, a study of the possible relationship between the composition of saliva and the condition of the teeth. From that time my interest professionally and scientifically in dental problems has been steadily cumulative.

In the course of these studies it soon became evident that one of the points of special contact between biological chemistry and dentistry was the dentifrice problem. It was obvious that the usefulness of a dentifrice, if any, was essen-

\footnotetext{
${ }^{1}$ William J. Gies is editor of the Journal of Dental Research and head of the biochemical department of Columbia University, College of Physicians and Surgeons.-Editorial note.
}

tially a physiological matter. Subsequently, we undertook a study of dentifrices from that point of view, and have been proceeding in an effort to comprehend the facts pertaining to the utility and quality of dentifrices.

It has long been considered essential that a dentifrice should be alkaline in order that it may neutralize the acid on the teeth when the dentifrice is applied to them. You understand, also, that practically all dentifrices, except mouth washes, contain solid material for the removal, by friction, attrition, or abrasion, of solid material from the teeth. The frictional agent and the neutralizing agent have commonly been regarded as the two essential features of the most useful dentifrices. To these have been added flavoring materials, to give the dentifrice an agreeable taste and power directly to stimulate the flow of saliva as an aid in the desired process of neutralization of "mouth acid."

A newer conception of the matter, and the one that I have personally been urging upon dental notice, and to which I ask your special attention, is a dentifricial method that would use an agent for the removal of dental plaques, tartar and debris, not only by friction, but also by a process that is essentially chemical in part, and which would not damage the enamel. We have found, in support of this idea, that the mucin plaque, the 
mucin film or adhesive on the teeth, can be broken and disintegrated and the mass disengaged, more effectively and more promptly, by the use of a suitably acid mixture than by any other means, and without damage to the teeth. A corollary to this newer view is the further conviction that the neutralization of the acid in the mouth by a dentifrice, at the moment when that dentifrice is applied to the teeth, is not the all-important hygienic consideration in this relation. The fact is that such neutralization of "acid mouth," if it is important, can be accomplished effectively by properly brushing the teeth with a suitably acid agent that causes alkaline saliva to flow profusely while the teeth are being brushed and for a short time thereafter.

In order that this general conception, which runs counter to general opinion and may seem to disregard experience, may not seem to be absurd, permit me to allude to one or two simple things that are apt to be overlooked or ignored in discussions of this problem.

All acids are compounds of hydrogen. Boric acid is a compound of hydrogen; sulfuric acid is a compound of hydrogen, and so on. If we wish conveniently to use a very general formula for acids, we might select $\mathrm{H}-\mathrm{R}$ to mean any acid, in which $\mathrm{H}$ stands for hydrogen and $\mathrm{R}$ for all the rest of that acid. Every acid may be represented, in principle, by that formula: H-R. It happens that when acids are dissolved in water they dissociate, with some exceptions, more or less completely into the $H$ part and the $R$ part, that is to say, into $\mathrm{H}$ and $\mathrm{R}$ ions. The acidic effect of a substance (dissolved in water) is an expression, in the main, of the influence of the $\mathrm{H}$-ions of that substance in the proportion involved. That fact is the key to understanding of all ordinary acidity effects in physiology or pathology.
One of the exceptional acids is boric acid. Dissolved in water, boric acid dissociates so slightly into $H$ and $R$ as to be practically undissociated. Boric acid, even in concentrated solution, can be put on the eyeball without damage to the eye-it can be applied to the eye, in saturated solution, with actual benefit to the eye under certain conditions! Think of putting vitriol on the eye, however! Sulfuric acid is a highly dissociable acid-yields H-ions freely; but boric acid is practically undissociable-yields H-ions very "reluctantly." When you think about effects of acids on the teeth and in the mouth, do not recall only the effects of concentrated sulfuric acid thrown on somebody's face, but think also of the effects of application of a saturated solution of boric acid to the eyeball as a common therapeutic expedient. Then you will realize the important difference between the two extreme types of acid and will see that there are "acids and acids." There are many gradations in dissociability-capacity to yield $\mathrm{H}$-ions--between these two extreme types of acids.

Acetic acid is another acid that dissociates only slightly. Even a 5 per cent solution of acetic acid, in water, is quite weakly acidic because only a small proportion of its hydrogen has gone into the ionic condition. Hydrochloric acid, on the other hand, whether it comes into the mouth from the stomach or with ferric chlorid from a bottle, yields its hydrogen readily in ionic form and is strongly acidic.

For these reasons one may deal with a "strong solution of a weak acid"- saturated boric acid solution, for exampleor with a "weak solution of a strong acid," such as 0.2 per cent hydrochloric acid in gastric contents; and vice versa. There is often confusion in this relation. One may have a "strong" (concentrated) solution of boric acid (a weak, poorly 
dissociable acid), which, put on the eye, will exert no deleterious influence; or a "weak" (dilute) solution of hydrochloric acid (a strong, richly dissociable, acid), which, on the eye, will have a bad effect.

Mucin is a weak acid that is insoluble in water. It is an acid protein and a protein acid. Let us use the letter $\mathbf{M}$ to represent that part of mucin that is not displaceable hydrogen. There are several displaceable hydrogen atoms in mucin. One general formula for mucin may be conveniently written, therefore, $\mathrm{HX}-\mathrm{M}$. Salivary mucin is a little stronger in acidic action, in some respects, than boric acid, but much weaker than acetic acid, or tartaric acid, or citric acid-common "food acids."

When salivary mucin is produced in the salivary glands, most of it is made to react there with such basic elements as sodium and potassium, and several compounds, mucinates, result. These mucinates are analogous chemically to the types of phosphate, as indicated by the two following illustrative formulas (for two sodium mucinates): $\mathrm{HX}_{\mathbf{X}^{-1}} \mathrm{Na}-\mathrm{M}$ and $\mathrm{HX}_{2} \mathrm{Na}_{2}-\mathrm{M}$. The hydrogen atoms are replaceable by one or more sodium atoms. The more sodium with which the mucin combines, for example, the more soluble is the compound, and vice versa. Mucin itself is insoluble in water. Saliva ordinarily contains a mixture of these types-soluble mucinate, and insoluble mucin and mucinate. Perhaps you are familiar with the fact that if you expectorate into water and stir up the mixture a fairly abundant supply of stringy material may be separated. The stringy substance is a mixture of mucin and mucinate containing a small proportion of base-all insoluble in the water. If you add alkali to saliva, the mucin and mucinate therein are converted into the mucinates containing relatively large proportions of base-all soluble in water. Such alkalized saliva mixed with water does not yield the stringy insoluble matter.

When saliva enters the mouth and bathes the teeth, the mucinous matter (mucin and mucinates) being more or less adhesive, sticks to the teeth in favored places, where friction does not dislodge it, and mucin films or plaques may be formed there. These masses, with bacteria that may be held in them and debris that may be added to them, become a visible and destructive feature on the surface of the teeth. Such films are difficult ordinarily to remove.

When one applies an alkaline solution, such as lime water, to a mucin plaque, alkali reacts with and enters the mass and makes a soluble calcium compound of the mucin-calcium mucinate. The mucinate swells and becomes viscid; but tho the mass is softened, it is not easily disengaged from its close contact with the surface because of its adhesiveness; altho in the continued presence of an excess of alkali, the film gradually dissolves. The bristles of a toothbrush, when the latter is applied to the teeth in the ordinary act of brushing, are apt to pass thru such a viscid film, after it is changed by alkali, without dislodging it; and the bristles may not completely remove the viscid mass, even by vigorous use of a brush. Anyone who has tried, with the aid of lime water or soap, and friction, to wash mucinous matter from a handkerchief containing nasal mucus knows how difficult it is to remove all the mucin from its attachments, under such conditions. The purpose to remove such mucinous material from the linen requires special effort when alkaline reagents are employed, apparently solely because of the adhesiveness of the viscid mucinate pro- 
duced from the mucin by the applied alkali.

Altho mucins dissolve in alkali, they do not do so readily under certain mechanical conditions, such as those pertaining to mucin plaques on teeth and nasal discharges in handkerchiefs. The conventional method of separating mucin from its alkaline solutions, that is, from solutions of mucin salts (mucinates), involves the addition to the alkaline solution of acid in sufficient quantity to impart a very slightly acid reaction to the fluid when the alkaline and acid portions are thoroly mixed. The acid thus added to the mixture, under such conditions, withdraws the base from the salt and produces the mucin acid, that is, the true mucin, which, being entirely insoluble in the medium thus created, is curdled, i. e., separates in flakes. These flakes are devoid of adhesive quality. The reaction involved in this flocculation may be indicated, in a general way, as follows:

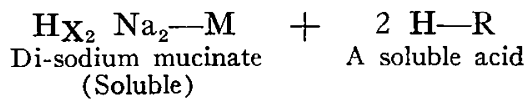

These facts imply that an effective way of removing a mucin plaque from a tooth, and mucinous matter from a handkerchief, would be the application of a medium that would impart to each a slightily acid reaction. The mucinate, in each case, would be curdled, flaked; it would lose its grip and could easily be flushed away. This is the chemical basis for my long-standing recommendation that "food-acid" media be used for the removal of dental mucin plaques. Some laundries use this acidic method to "wash" mucinous matter out of linen.

If these statements fully represent the facts, and if acid media can be included in dentifrices without damage to the teeth, you would naturally conclude that the use of suitably acid dentifrices would satisfactorily accomplish complete removal of dental mucin plaques, which you regard as important mechanical factors in the etiology of dental caries. Such dentifrices, you would agree, would be particularly useful agents in the art of keeping teeth clean, provided they did not harm the teeth.

I presume you will be inclined to believe that the chemical facts I have presented are, in principle, merely conventional biochemical knowledge. I cannot expect you to believe, however, that acid media may be applied to the teeth as dentifrices without damage to the teeth, unless essential facts are summarized in this relation. Permit me to present some of the "essential facts."

Paleontologists agree that man has ascended from a succession of species of animals that have fed very largely on fruits, all of which were acid in reaction. There is a fair presumption, accord-

$=\underset{\substack{\text { Mucin } \\ \text { (Insoluble) }}}{\mathrm{HX}-\mathrm{M}}+\underset{\text { A soluble salt }}{2 \underset{\mathrm{NaR}}{\mathrm{N}}}$

ingly, that the ingestion of acid foods, thru the ages, has not materially damaged the teeth of the so-called average individual in each successive race from which man has ascended, nor of man himself.

Neariy all the masses of food we eat are acid in reaction, some of them, such as fruits and ordinary salads, being quite decidedly so. Anthropologists have reported that the teeth of fruit eating savage races, and of such groups of civilized people as the Sicilians, who eat large amounts of citrous fruits, are uncommonly fine in all respects.

Many of the lower types of animals feed largely on fruits (all of them acid) 
without discernible detriment to the teeth as a consequence, and certainly without practical damage to the teeth.

Fruits such as lemon and orange, acid always, are among the types of food that prevent scurvy, and facilitate the development and maintenance of normal dentition - certainly not deleterious effects.

Natural extracted human teeth, containing typical fillings of various kinds and set in "artificial jaws" of paraffin, were brushed twice daily for five years and one day (1913-18) with a mixture of equal parts of water and vinegar, without causing any damage to the enamel that could be detected by a committee of dentists of the First District Dental Society of the State of New York. (The vinegar-water mixture was selected for this test because it represented a degree of "food-acidity" that would probably be regarded generally as "too high for safety." It was felt that the "food-acid theory" should be tested with a mixture close to the maximum degree of food acidity rather than near the minimum, on the assumption that, if an unnecessarily or undesirably high degree of acidity did no harm, all lower degrees of acidity would assuredly be harmless.)

The most acid foods, such as fruits, are the most active stimulants of the secretion of saliva containing high degrees of alkalinity and low proportions of mucin and mucinates. The ingestion of such acid foods is followed by an "after-flow," to some degree, of saliva of high degrees of alkalinitygreater power to neutralize acid!

The juices of such mildly acid fruits as apples are strong enough to curdle and dislodge dental mucin plaques. Who will contend that the ingestion of apples as food is undesirable for any dental reason?

Undiluted grape-fruit juice may suit- ably be, and is very commonly, eaten with a spoon. Why may not such juice be applied, diluted especially, to the teeth with a toothbrush?

Does any dentist object to the drinking of carbonated waters because they are acid in reaction?

I believe I am fully justified in stating the conviction that, if the mucin plaque is a mechanical factor in the etiology of dental caries, the removal of such plaques may be satisfactorily effected, chemically, physiologically and hygienically, by the ordinary use of suitably selected dentifrices containing "food-acids" in proportions lower than those in fruits such as oranges.

For twelve years I have been presenting this opinion to dentists with the suggestion that the idea be tested clinically by dentists. I have declined to exploit the idea or to derive any financial advantage whatever from its evaluation by others. I appeal to you to test the matter for yourselves individually, with ordinary food-acid media in simple ways to suit your own ideas in the matter of procedure.

Several years after I made the suggestion that "food-acid" media are desirable constituents of dentifrices, Pickerill published the same proposal. We arrived at the same conclusion, but by different routes and for different reasons. Pickerill ascribes the advantage derivable from the use of acid dentifrices to the stimulating influence of such acid media on the production of alkaline (acid-neutralizing) saliva. My own idea has been that the benefit accruing from the use of a suitably acid dentifrice is primarily due to direct disintegrative action on mucin plaques and only secondarily (tho importantly, too) thru the long-known stimulative influence of acidity on the secretion of saliva.

There appears to be a growing tend- 
ency, in the dentifrice trade, to adopt the principle underlying the views Pickerill and I have published. This "growing tendency" seems to be the result of increasingly favorable dental opinion of available dentifrices compounded on the "food-acid theory."

Before concluding I ought to refer to several other important phases of this subject, to prevent possible misapprehension.

The direct attacking and destructive agent in superficial enamel decay-in the initial phase of caries-is acid produced by bacteria, supposedly lactic acid. (Lactic acid is one of the "food-acids"a constituent of sour milk, for example.) How would an acid dentifrice prevent or retard the action of lactic acid and similar acids produced on dental surfaces by bacteria? I answer: By completely removing the mucin plaques and debris in which bacteria are actively engaged in producing lactic acid and similar acids, and also simultaneously by washing away any accumulation of such acids. This would be accomplished as often as the dentifrice would be effectively used; and, by the completeness of the removal of the plaque, would particularly delay the development of new destructive centers of fermentation on the teeth.

If lactic acid, or similar acid, is the destructive agent in the initiation of caries, why does not the acidity of the various fruits also damage the enamel? Again I reply: In the initiation of caries the acid is produced molecule by molecule continuously at a given point, whereas the presentation of "food-acid," in food or dentifrice, does not involve continuous production and replacement of active acid molecules at any point on a tooth. "Water dropping day by day will wear the hardest rock away," but the same amount of water poured over a rock by the barrel would merely wash the rock.

If "food-acid" is useful in a dentifrice, why does not the mere ingestion of fruit keep the teeth clean? I believe the regular ingestion of fruit-apple, for example-does keep clean the dental surfaces that are freely swept by the fruit juice and particles. The juice from ingested fruit, however, is rarely if ever passed effectively over all the dental surfaces by the process of mastication or otherwise. Besides, fruit particles containing highly fermentable matter tend to lodge on and between the teeth, and thus may favor fermentation and local production of acid. A dentifrice of the same kind and degree of acidity, however, would contain no such fermentable matter and would be effectively applied (?) as a cleanser.

Finally, what may be expected of any dentifrice if it is not used often enough, or is not applied effectively enough to the surfaces of the parts of the teeth on which fermentable matter lodges or is retained? What is the therapeutic value of the best medicine while it remains in a bottle or when it is thrown to the floor? Can the face be kept clean by frequently and thoroly washing only the hands with soap and water?

Has the mechanical process of applying dentifrices attained the degree of perfection that is required by the necessities of successful oral hygiene and by the ideals of preventive dentistry? This question, to which I believe our unanimous answer is no, carries me to and beyond the edge of my "problem."

\section{Discussion}

E. D. Coolidge, Chicago Illinois: Dr. Gies is a scientific man who is studying this problem in the research laboratory and is giving us information that will place us in a position not to be misled by the various advertisements for dentifrices. The manufacturers pick up new ideas from dental literature and 
place them in large type on their circulars as they have done with the acid or pepsin theory. They are just as apt to put something in print that will mislead the dentist as the public, and in this way derive a great deal of benefit financially from an unsuspecting public; in other words, they capitalize ideas that are founded on facts established by scientific investigation but their product which they advertise is oftentimes valueless.

The character of the saliva I believe is of very great importance in mouth hygiene. The character of the saliva is affected by the food we eat, especially by fruit acids, whether in the form of a dentifrice or by the fruit itself. I have been converted to that for many years and have tried it in my own life and have found that it gives results. The character of the saliva is not affected only by fruit acid as it relates to oral hygiene, but may be affected by other things that we eat. The natural tooth wash is saliva. The more viscid the saliva, the more difficult it is to remove the deposits from the teeth, the more the deposits will settle upon the teeth and the greater the need for mechanical friction to maintain mouth hygiene.

I believe Dr. Gies made a statement of the greatest importance when he spoke of the necessity of getting a dentifrice upon the tooth, but not so much from the standpoint of the dentifrice dissolving the bacterial plaque upon the tooth as from the act of getting it on the tooth and the rubbing that will mechanically break up the mucinous plaque and remove it.

$\mathrm{He}$ has given us the chemistry of the formation of the plaque, and has told us that organic acid will make it much more easy to remove. There is no question but that this fact is important. But let us not forget the fact that mechanical friction is the important thing in removing material from the teeth. The character of the saliva has a great deal to do with the difficulty of removing these plaques. It has also a great deal to do with the amount of deposit that forms upon the teeth and the need for that friction. By improper diet it is possible to make it necessary to brush the teeth frequently to keep them clean, while it is also possible to be able to keep the teeth in a very clean condition with considerably less brushing if the diet is properly regulated in quantity as well as in quality. The amount of deposits upon the teeth may be considered a barometer which indicates an excess of diet. The deposits must be kept off from the teeth to maintain mouth hygiene, because the latter depends not only upon freedom from dental decay but also upon gingivitis, and deposits of calculus are a yery important factor.

Arthur G. Smith, Peoria, Illinois: This rematkably searching, clear-cut, contribution is one of great interest to all of us. While one swallow does not make a summer, nor one "history" a rule, I cannot refrain from citing a case which I think will be of interest to all of you and to Dr. Gies.

I had a patient sixty years of age, whose teeth were in a remarkably fine condition. No dental caries had appeared in his mouth for forty years and his teeth were absolutely free from deposits. I asked this man as to his dietary habits. He said: "I eat anything I can overtake, but I suppose I do one thing that is a little out of the ordinary. Every night before I go to bed, after I brush my teeth, I eat two oranges if they are large ones, and four if they are small ones."

Otto U. King, Chicago, Illinois: There are few persons now who have not the conviction that the scientific cleansing of the teeth and gums is very valuable, and the business in antiseptic dentifrices is obviously increasing, judging from the numerous public advertisements which appear in the daily press setting forth the merits of a great number of these preparations. Generally speaking, they are so composed as to render them well adapted for the purpose, and we have subjected a number of them to analytical examination. The choice of antiseptic is commonly confined to carbolic acid, thymol, peppernint, boric acid, benzoic acid, eucalyptus and other essential oils, distributed in vehicles containing as an agent of attrition non-gritty chalk and as an alkaline medium soap. Experiments are usually quoted as to the actual germicidal value of these preparations in vitro, but, of course, it would be more satisfactory to have evidence of their real effect in the mouth, where very different conditions must, as a matter of fact, exist. The use of a tooth wash does not approach the conditions of a laboratory test, tho there can be little doubt that a great deal of germicidal work in the mouth is done by the vigorous application of the toothbrush, and it may be pointed out that the tongue may well be included in the process. To be effective, however, the action of all antiseptics takes time, according to the vitality of the organisms they encounter, and usually the toothbrushing process does not occupy many seconds. The question of time-exposure is important, but it is very generally overlooked, and conseruently the antiseptic treatment of the teeth falls short of that effectiveness which is shown to be the case in laboratory experiments. The toothwashing process should be more prolonged, and the antiseptic wash allowed to remain in contact with the teeth and gums for some minutes, instead of seconds, before finally washing the mouth clear of antiseptic with plain water.

It is a matter, perhaps, for discussion as to whether it is desirable to use tooth pastes 
or washes which are very strongly bactericidal. The objections offered may be summed up in two statements, namely: (1) that substances which would destroy bacteria injure the mouth mucous membrane; (2) that many mouth bacteria are normally found there, and their activities are probably of some importance in the preparation of food for digestion, and these bacteria would be destroyed along with the pathogenic bacteria. Whether these statements are true or not, there occur in the advertising of tooth pastes and mouth washes frequent claims as to the bactericidal power of these preparations.

On this account it seemed wise to make tests which might confirm or disprove such statements. Mouth bacteria were taken from badly infected mouths, selected from patients presenting at the Northwestern University Dental School Clinic. ${ }^{2}$ Suspensions were made of these bacteria in salt solution and the inhibiting power of tooth paste emulsions tested on cultures made from these suspensions. The too:h paste emulsions were added to the cultures so that their action was not confined to five or ten minutes of time, but thruout the twenty-four to forty-eight hours that the cultures were incubated.

While it is true that they were used in the strength of only 1 per cent of the original paste in the culture plates, i.e., 1 c.c. of a 10 per cent emulsion in 10 c.c. of culture media, yet the bacteria were exposed to the inhibiting action of the emulsion from twentyfour to forty-eight hours, as stated.

This work, to be reported, is merely preliminary to a study of the inhibitory action of the tooth paste emulsion in other dilutions and with all factors arranged to correspond to conditions which obtain in the average cleaning of the teeth.

Final conclusions can only be arrived at when the bacteria are exposed to comparatively high concentrations of the tooth paste for five to ten minutes. But after testing the inhibitory action of ten different tooth pastes purchased at random from various drug stores, it may be said that in these low dilutions the growth of mouth bacteria is not materially retarded.

By adding litmus solution and lactose to the culture plates it was possible to demonstrate also that the growth of the lactic acid corming bacteria was not retarded.

Out of these ten plates there were three in which it was possible to say that in the plates containing the tooth paste emulsions the

\footnotetext{
${ }^{2}$ Experimental work conducted at Northwestern University Dental School Clinic and Research Laboratory, by $O$. R. Buttner, D.D.S., under the direction of E. H. Hatton, M. D.
}

bacterial colonies were smaller and slightly less numerous than the ones in the control plates, and one in which the culture media immediately turned pink on the addition of the tooth paste emulsion because of its acidity.

Bacteria grow in an alkaline media; therefore an acid substance would inhibit their growth.

Dr. G. V. Black contended that we were approaching the time when we can expect to produce an immunity from caries either from physiological conditions or from treated conditions in the saliva itself.

Dr. Joseph P. Michaels, Paris, in his SialoSemeiology, says that if there is a balance (not artifically produced) of sulphocyanide of potassium and ammonia in the saliva, there will be no caries. Up to the present time it has been impossible to produce this immunity artificially.

In diphtheria the injection of an antitoxin will prevent the pathogenic activity of the diphtheria bacillus in the individual. Autogenic vaccines will eliminate from the mouth pus-producing germs for certain brief periods of time. If that can be produced artifically, why cannot a similar immunity be produced in regard to caries of the teeth?

Therefore the real scientific research problem confronting the dental profession today is the problem of securing an immune condition from caries in the saliva.

In conclusion I want to stress this point, namely, I am not ready to admit thit there may not be some real virtue in too h paste or powder containing anti-substances. If we have inhibited the growth of bacteria in the saliva for even a brief period of time, it would seem that we have at least started on the road to immunity from caries of the teeth. Scientists who are trying to solve the problem of prevention of dental caries by any methods should be encouraged and such men should receive the moral and financial support of the dental profession.

Arthur D. Black, Chicago, Illinois: I would like to mention four things which we should keep definitely in mind in connection with this subject: (1) Acidity of the saliva has absolutely nothing to do with decay of the teeth. (2) It is only that acid that is held in contact with the teeth under the gelatinous plaques which causes the teeth to decay; the gelatinous plaque prevents this acirl from becoming mixed with the saliva. (3) Antiseptic mouth washes have no value whatever in preventing decay of the teeth or in bringing about immunity. (4) Certain acids placed in the mouth have a tendency to inhibit the action of lactic acid-forming micro-organisms, and also a tendency to dissipate the plaques.

It will be noted from these statements that 
any substance taken into the mouth which would dissolve the gelatinous plaques, thus permitting the lactic acid to become mixed with the saliva, would tend to prevent decay.

Dr. Gies (closing): May I allude briefly to one or two items in Dr. Coolidge's remarks? One is the difficulty of removing mucin plaques by friction. I referred to the mechanical difficulty of getting mucinous matter out of linen with the aid of alkalies. It is a well known fact that relatively great force is required under such circumstances. With the acidic treatment, however, the mucin may be removed without trouble. The acid flakes the mucin and the flakes, having no adhesiveness, may be readily flushed away.

Dr. Coolidge's remarks about the effect of various things on the secretion of saliva suggest additional comment. The kind of substance which, taken into the mouth, makes saliva least mucinous, most watery, most alkaline, and nearest to what is supposed to be the phy'siological ideal, is "food-acid," such as that in ordinary fruit.

There is nothing you can take into the mouth that makes saliva as thick, as mucinous, or as adhesive as sugar. Abundance of sugar in the diet is believed by many physiologists to account for much of the progress and apparent cumulative increase of dental caries among civilized peoples, for sugar not only makes the saliva thick and favors formation of mucin plaques, but it also provides a suitable pabulum for localized bacterial production of acid. If candy is eaten before retiring, and any of it remains in the mouth, a considerable degree of acidity may be produced and localized during the night-and appre- ciable harm to the teeth, "in spots," may thus be registered during a period of six to eight hours.

Dr. Black's father suggested the use of purgatives to prevent deposition on the teeth. Newer findings emphasize the significance of that expedient. Intestinal putrefaction and fermentation result in the production of solvents of calcareous material in the large intestine, and tend to increase the proportion of calcareous substances in the blood. The salivary glands are very active in excreting such substances into the mouth.

We know that hard tartar is deposited largely because carbonic acid is discharged from the soluble bicarbonate in the saliva. The saliva becomes more alkaline as the carbon dioxide is discharged from the bicarbonate, and calcium carbonate and phosphate tend to be precipitated simultaneously. A relatively abundant salivary excretion of bicarbonate and phosphate of calcium, for any reason, and discharge of carbon dioxide after the secretion emerges in the mouth, induce relatively heavy deposition of the ingredients of hard tartar. The activity of the salivary glands, and their tendency to excrete much or little of these substances, are plainly involved.

I cordially endorse what Dr. Coolidge has said on the destiny of oral hygiene: the problem is not merely one of means of removing from the teeth something already deposited there of unhygienic tendency, but also one of methods of preventing development of unhygienic dental and oral conditions. Sooner or later the latter and more important phase will receive the scientific and experimental attention it assuredly deserves. 